\title{
Serial Patch Array Antenna for an FMCW Radar Housed in a White Cane
}

\author{
Stefano Pisa, Erika Pittella, and Emanuele Piuzzi \\ Department of Information Engineering, Electronics and Telecommunications, Sapienza University of Rome, Rome, Italy \\ Correspondence should be addressed to Stefano Pisa; pisa@diet.uniromal.it
}

Received 15 August 2016; Accepted 20 September 2016

Academic Editor: Yu Jian Cheng

Copyright (C) 2016 Stefano Pisa et al. This is an open access article distributed under the Creative Commons Attribution License, which permits unrestricted use, distribution, and reproduction in any medium, provided the original work is properly cited.

The design, realization, and test of the transmitting and receiving antennas for an FMCW radar operating between 24 and $24.25 \mathrm{GHz}$ (ISM band), to be housed in a white cane for improving mobility of visually impaired subjects, have been performed. The coaxialto-microstrip transition necessary for feeding the antennas has been studied together with the characteristics of serial arrays of increasing number of patches. Based on this study, a serial array with 8 patches has been selected for the transmitting and receiving antennas. The realized structures show a reflection coefficient lower than $-10 \mathrm{~dB}$ in the ISM band. When the antennas are attached to an FMCW radar board, the radar is able to record reflections from a metallic panel up to a $5 \mathrm{~m}$ distance.

\section{Introduction}

The mobility in unknown environments is one of the major problems for visually impaired people [1]. Usually they employ aids such as the white cane or the guide dog [2]. However, these solutions are not optimal. For example, the more widespread white cane has only a limited exploration capability, providing no information on obstacles at the upper body height and on the obstacle's actual distance, size, and possible movement.

Recently, some electronic travel aids (ETAs) for visually impaired people's mobility have been developed $[3,4]$. They are based on ultrasonic or optical systems whose primary function is the detection and location of objects along the path of the user. However, such systems present some limitations, such as the limited range, the difficulty to operate in the presence of smooth surfaces with a low incidence angle. Recent studies showed the possibility of using electromagnetic radiation for the detection of obstacles placed in front of visually impaired people $[5,6]$.

Ultrawideband radars can be used for realizing ETA devices. These radars have high resolution [7] but require bulky antennas $[8,9]$. On the contrary, frequency modulated continuous wave (FMCW) radars operating from 24 to 24.25
$\mathrm{GHz}$ (ISM band) can use compact planar antennas. Moreover, thanks to its small dimension, an FMCW radar can be easily housed in the white cane.

In this paper, the design of the transmitting and receiving antennas of a $24 \mathrm{GHz}$ FMCW radar to be housed in a white cane has been performed. Among the various radiating structures, serial patch arrays have been selected. These antennas have been studied together with their feeding structure. Then, the selected antennas have been realized and their return loss has been measured. Some preliminary radar tests have been also performed.

\section{FMCW Radar}

The schematic of the considered FMCW radar is reported in Figure 1(a). The main block of the radar is constituted by the BGT24MTR11 integrated circuit (IC) by Infineon (see Figure 1(b)). This IC exploits a voltage-controlled oscillator (VCO, Figure 1(a)) operating between 24 and $24.25 \mathrm{GHz}$ (ISM band). The power delivered to the radiating antenna by the power amplifier (PA) is about $11 \mathrm{dBm}$. The receiving channel is constituted by a low noise amplifier (LNA) and an I\&Q demodulator. 


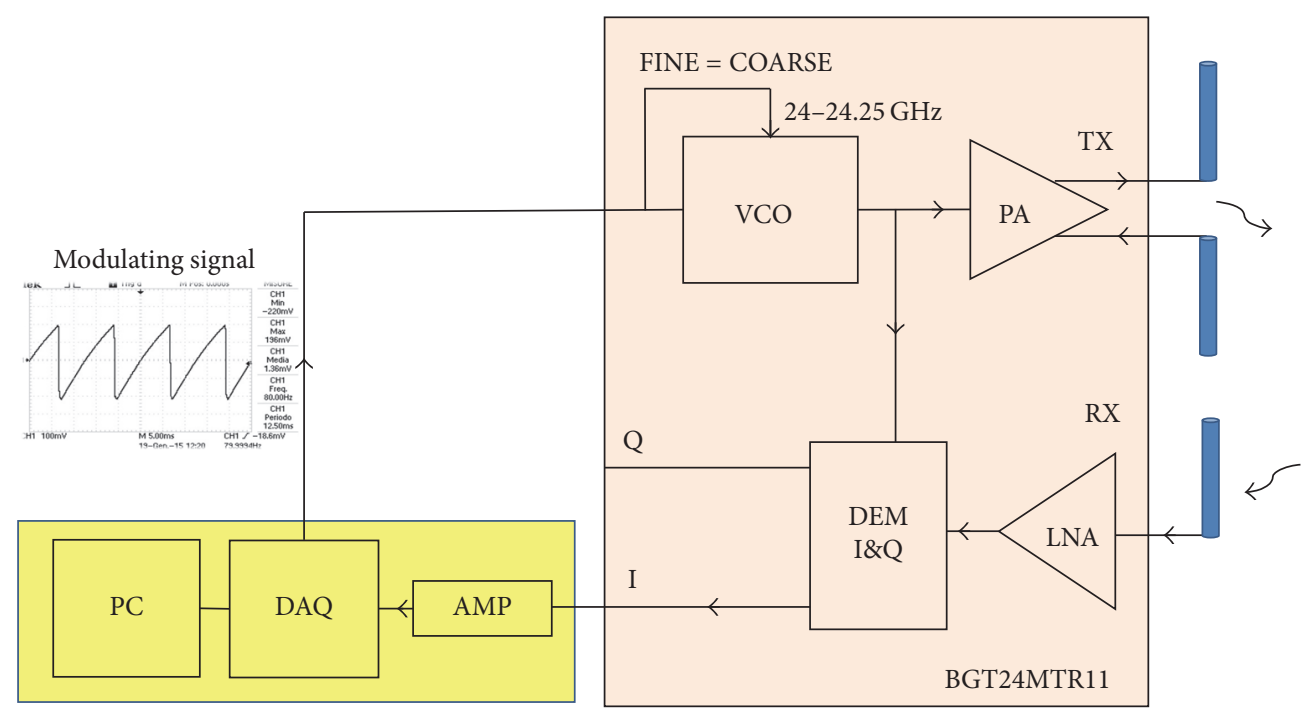

(a)

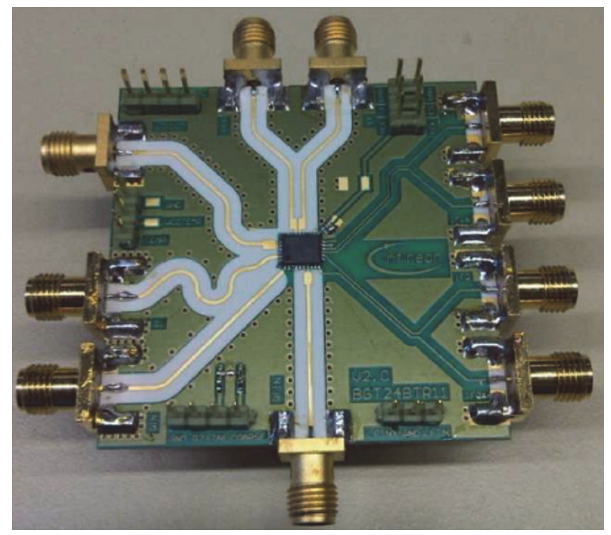

(b)

FIGURE 1: Schematic of the considered FMCW radar system (a) and photo of the BGT24MTR11 integrated circuit by Infineon (b).

From the FMCW radar theory [10], the range information $(R)$ is converted into an output signal frequency through the following equation:

$$
f_{\text {Delay }}=\frac{2 R}{c} \frac{\Delta f}{T}
$$

where $c$ is the speed of light, $\Delta f=250 \mathrm{MHz}$ is the radar bandwidth, and $T$ is the repetition period. Moreover, the radar distance resolution is

$$
R_{D}=\frac{c}{2 \Delta f}=0.6 \mathrm{~m}
$$

For an antenna with azimuthal $-3 \mathrm{~dB}$ aperture equal to $\theta_{A}$, the radar lateral resolution is

$$
R_{L}=2 R \sin \left(\frac{\theta_{A}}{2}\right) .
$$

Figure 2 shows a scheme of the radar housed on the white cane together with the position of possible obstacles.
In order to guarantee a field of view between two and five meters with $0.8 \mathrm{~m}$ lateral resolution, an antenna with $\theta_{A}$ of about $10^{\circ}$ will be necessary. Moreover, since the radar output (TX, Figure 1(a)) is differential, a balanced transmitting antenna will be designed.

\section{Transition and Antenna Models}

3.1. Coaxial-to-Microstrip Transition. Since radiofrequency inputs and outputs of the BGT24MTR11 IC are coaxial SMA connectors (see Figure 1(b)), in order to use microstrip fed serial patch array antennas, a coaxial-to-microstrip transition has to be designed. The usual approach to feed planar antennas consists in using SMA edge-mounted connectors whose central pin is directly soldered to the microstrip. However, due to the high frequency employed by the FMCW radar, this approach results in a significant mismatch and different solutions have to be used.

In general, a well-designed transition converts the transverse field configuration and characteristic impedance of one 

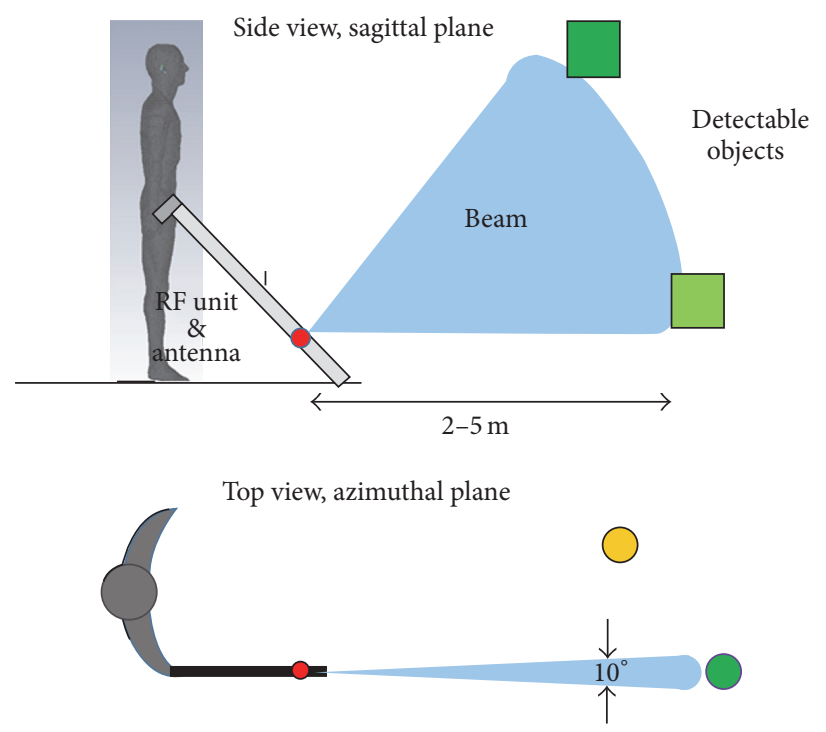

FIGURE 2: Scheme of the considered scenario.

transmission line to that of the other transmission line over a desired frequency band of operation while maintaining low insertion loss and high input return loss. Unfortunately, the coaxial and microstrip line fields are very different. Since the field cannot change instantaneously, a transition zone is created, in which additional unwanted field components are generated. In turn, this can give rise to both parasitic and radiative behaviors. These effects must be minimized in order to keep the insertion loss factor under acceptable values.

In this paper, to solve this problem, the geometry shown in Figure 3 has been suggested.

The structure consists in the cascade of three waveguides: a coplanar waveguide (CPW), a grounded coplanar waveguide (GCPW), and a microstrip (MS). The basic idea of this structure is to let the field change smoothly keeping the characteristic impedance always equal to $50 \Omega$ over all the sections. Moreover, realizing a CPW waveguide in the very first part of the track makes the field distribution closer to a coaxial line configuration and allows for easy soldering of the central pin of the coaxial SMA connector, since the central line of the CPW is wide enough.

Even though the microstrip and the CPW are both planar waveguides, their field is different. A better field matching is achieved inserting a GCPW between the CPW and the microstrip. In this manner, the EM field slowly changes to a GCPW field up to become MS field [11].

In the GCPW-to-microstrip transition, the two ground planes can be linked through a via hole. However, as shown in $[11,12]$, via-less transitions can enlarge the frequency bandwidth. Therefore, in this realization, no vias are used and the ground plane of the microstrip and the ground pads of the CPW are electromagnetically coupled.

For the physical realization of the radar antennas, the same substrate suggested in the BGT24MTR11 datasheet for the device mounting has been used. This is a $10 \mathrm{mil}(254 \mu \mathrm{m})$ thick Rogers RO4350B laminate with a dielectric constant of 3.66 , a loss tangent of 0.004 , and a copper thickness of $35 \mu \mathrm{m}$.
The transition dimensions have been designed by using the program TXline available inside the NI AWR Design Environment. After setting the substrate parameters, the tool yields microstrip line impedance of $50 \Omega$ with $\mathrm{WC} 3=527 \mu \mathrm{m}$ and $\mathrm{GAP} 3=800 \mu \mathrm{m}$. For the $\mathrm{CPW}$, the tool gives $\mathrm{WC1}=$ $1.5 \mathrm{~mm}$ with GAP1 $=120 \mu \mathrm{m}$.

The dimensions of the hybrid GCPW that stands in between these two have been evaluated according to [12]. That means

$$
\begin{aligned}
\mathrm{WC} 2 & =\frac{\mathrm{WC} 3+\mathrm{WC} 1}{2} \\
\mathrm{GAP} 2 & =\frac{\mathrm{GAP} 3+\mathrm{GAP} 1}{2} .
\end{aligned}
$$

The width (WC) of the aperture in the ground has been realized according to the shape proposed in [12], while the total length of the transition $L$ has been split in partial lengths according to

$$
L_{1}=L_{2}=\frac{L}{2} .
$$

Afterwards, the width WC and the length $L$ have been optimized with parametric simulations performed by using the CST Microwave Studio software. The best values found were $L=1 \mathrm{~mm}$ and $\mathrm{WC}=\mathrm{WC} 1+0.2 \mathrm{~mm}$.

3.2. Serial Array. By using the classical approach described in [13], the design of a rectangular patch operating at $24.125 \mathrm{GHz}$ has been performed, resulting in the following patch length: $L_{p}=3.14 \mathrm{~mm}$. Hence, a square patch with this side fed by a $50 \Omega$ transmission line $\left(W_{l}=0.4 \mathrm{~mm}\right)$ with $\lambda / 2$ length $\left(L_{l}=3.7 \mathrm{~mm}\right)$ has been studied by using the CST software (see Figure 4(a)).

Figures 4(b) and 4(c) show the distributions of $E_{z}$ and $H_{z}$ fields on the patch $(x-y)$ plane for a radiated power of $500 \mathrm{~mW}$. As expected for a microstrip patch, the maximum of the 

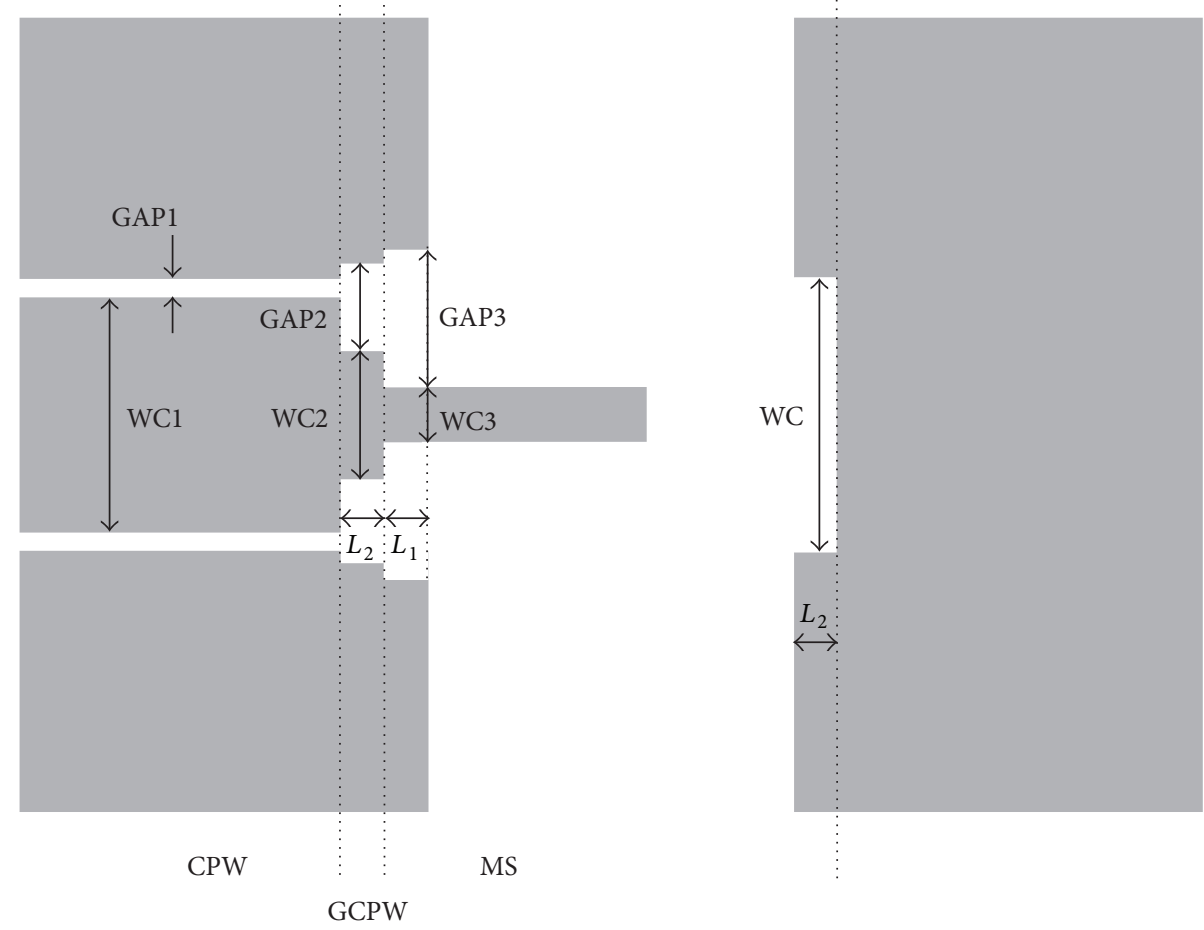

(a)

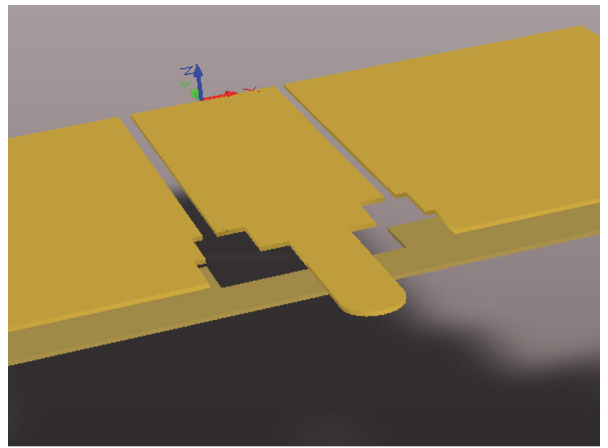

(b)

FIGURE 3: CPW-to-microstrip transition dimensions (a) and 3D footprint (b).

electric field is present at the near and far ends of the structure with respect to the feeding line, while the magnetic field is stronger at the left and right sides. Figures 4(d) and 4(e) show the radiation pattern on sagittal $(x-z)$ and azimuthal $(y-z)$ planes. In the sagittal and azimuthal planes, $-3 \mathrm{~dB}$ aperture angles are $86.3^{\circ}$ and $104^{\circ}$, respectively, while the directivity is $6.8 \mathrm{dBi}$. The input impedance $\left(Z_{\text {in }}\right)$ is about $310 \Omega$. All these values are very close to the theoretical expectations for a square patch [13].

After that, serial arrays with an increasing number of patches have been studied. As an example, Figure 5(a) shows the geometry of a serial array with 8 patches and Figures 5(b) and 5(c) show $E_{z}$ and $H_{z}$ field distributions on the patch plane $(x-y)$. The spatial regularity of these distributions which is an indication of the correct design of the structure is worth noting. Figures 5(d) and 5(e) show the simulated radiation patterns on the sagittal $(x-z)$ and azimuthal $(y-z)$ planes, respectively. As can be noted, the sagittal plane pattern is quite similar to that of the single patch, while a strong reduction of the aperture in the azimuthal plane can be observed. For this structure, in the sagittal and azimuthal planes, $-3 \mathrm{~dB}$ aperture angles are $78^{\circ}$ and $11^{\circ}$, respectively, while the directivity is $15 \mathrm{dBi}$. The input impedance $\left(Z_{\text {in }}\right)$ is about $56 \Omega$.

Table 1 shows the results for all the studied configurations. From the table, it can be observed that increasing the number of patches results in a reduction of the radiation impedance towards the desired $50 \Omega$ value, with a corresponding reduction of the reflection coefficient. When more than 6 patches are considered, the real part of the radiation impedance still reduces but, due to the growth of the imaginary part, the reflection coefficient presents a small increase. Concerning the radiation pattern, by increasing the patch number, a 


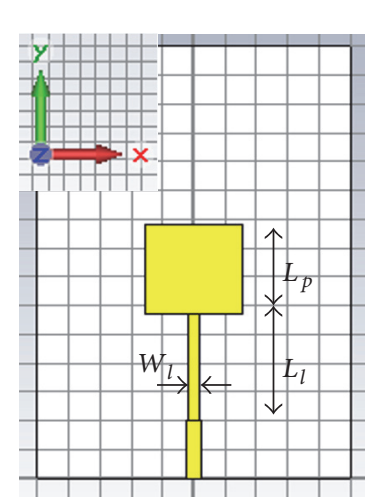

(a)

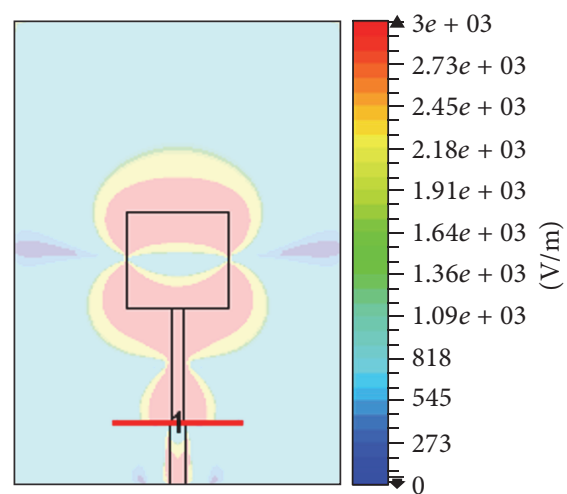

(b)

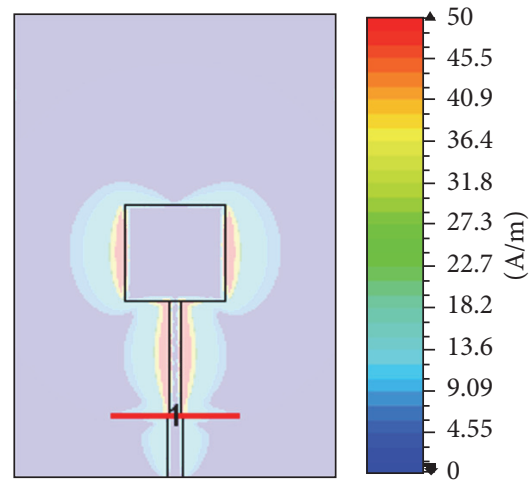

(c)

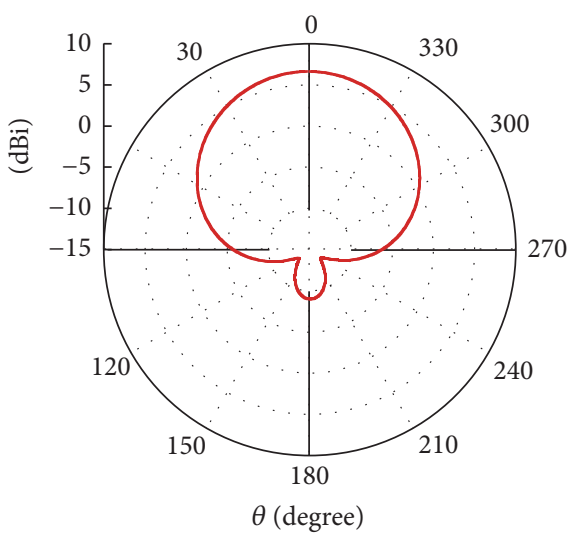

— Farfield directivity Abs $(\phi=0)$

(d)

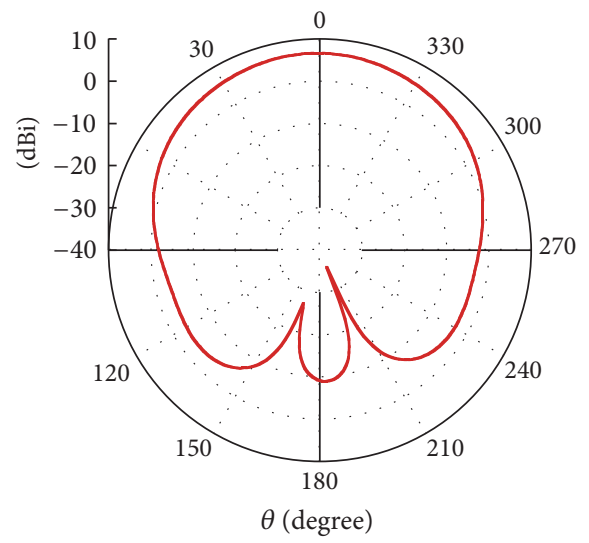

- Farfield directivity Abs $(\phi=90)$

(e)

Figure 4: Single-patch geometry (a); $E_{z}$ field distribution (b) and $H_{z}$ field distribution (c) on the patch plane $(x-y)$; sagittal $(x-z)(\mathrm{d})$ and azimuthal $(y-z)(\mathrm{e})$ radiation patterns.

reduction of the antenna aperture in the azimuthal plane $\left(\theta_{A}\right)$ and a corresponding increase in the directivity $(D)$ can be observed.

3.3. Transmitting and Receiving Antennas. In order to guarantee a field of view between two and five meters with a lateral resolution of about $0.8 \mathrm{~m}$, an antenna aperture in the azimuthal plane of about $10^{\circ}$ is necessary (see (3)). Hence, a serial array with 8 patches was chosen for the transmitting and receiving antennas. In particular, since the radar output (TX in Figure 1(a)) is differential, a balanced transmitting antenna has been realized (see Figure 6(a)). Figures 6(b) and 6(c) show $E_{z}$ and $H_{z}$ field components, and Figures $6(\mathrm{~d})$ and $6(\mathrm{e})$ show the simulated radiation pattern on sagittal $(x-z)$ and azimuthal $(y-z)$ planes, respectively. From Figure $6(\mathrm{e}),-3 \mathrm{~dB}$ aperture in the azimuthal plane of about $10^{\circ}$ is evidenced together with maximum directivity of about $14 \mathrm{dBi}$.

As concerns the receiving antenna (see Figure 7), it uses two four-patch serial arrays attached to a Wilkinson power combiner whose two outputs have a phase difference of $180^{\circ}$ with each other. This structure is preferable with respect to that in Figure 5(a) due to its improved symmetry as evident by comparing Figure 5(e) with Figure 7(e). The obtained radiation patterns are very similar to those of the transmitting antenna.

\section{Transition and Antenna Realization}

4.1. Transition Realization. To test the designed coaxialto-microstrip transition, it has been assembled inside the CST program together with a five-patch serial array (see Figure 8(a)). Then the structure has been converted in Gerber format and imported inside the ISOPRO program. This software is able to control the Quick Circuit system distributed by T-Tech ${ }^{\circledR}$, which is a fast PCB printer able to mill copper and to create the tracks on a board (see Figure 8(b)).

After the coaxial-to-microstrip transition realization, a second substrate (Arlon AN305) has been stuck under the antenna using commercial glue, and the SMA connectors have been soldered. This additional substrate is used in the evaluation board provided by Infineon to realize additional connections. In this single-layer release, the second substrate has the only purpose to increase the robustness of the structure. In fact, with only $254 \mu \mathrm{m}$ thick substrate, the board 
TABLE 1: Synthesis of simulation results for serial array with 1 to 8 patches.

\begin{tabular}{|c|c|c|c|c|c|c|c|}
\hline $\begin{array}{l}\text { Number of } \\
\text { patches }\end{array}$ & $\begin{array}{c}\operatorname{Re}\left(Z_{\mathrm{IN}}\right) \\
(\Omega)\end{array}$ & $\begin{array}{c}\operatorname{Im}\left(Z_{\mathrm{IN}}\right) \\
(\Omega)\end{array}$ & $\begin{array}{c}\left|Z_{\mathrm{IN}}\right| \\
(\Omega)\end{array}$ & $\begin{array}{l}\left|\Gamma_{\mathrm{IN}}\right| \\
(\mathrm{dB})\end{array}$ & $\begin{array}{c}\theta_{S} \phi=0 \\
\left({ }^{\circ}\right)\end{array}$ & $\begin{array}{c}\theta_{A} \phi=90 \\
\left({ }^{\circ}\right)\end{array}$ & $\begin{array}{c}D \\
(\mathrm{dBi}) \\
\end{array}$ \\
\hline 1 & 310.52 & 23.52 & 311.41 & -3.54 & 83.6 & 104.3 & 6.8 \\
\hline 2 & 188.24 & 74.44 & 202.42 & -5.09 & 79.0 & 42.7 & 10.3 \\
\hline 3 & 113.80 & 73.05 & 135.23 & -6.65 & 80.3 & 31.2 & 11.2 \\
\hline 4 & 94.89 & 57.43 & 110.91 & -8.14 & 78.8 & 21.8 & 12.9 \\
\hline 5 & 76.93 & 0.48 & 76.93 & -20.02 & 78.0 & 17.8 & 13.7 \\
\hline 6 & 68.06 & 8.25 & 68.56 & -22.63 & 79.0 & 15.1 & 14.3 \\
\hline 7 & 56.55 & 21.77 & 60.59 & -14.57 & 78.6 & 12.7 & 14.7 \\
\hline 8 & 56.27 & 27.29 & 62.49 & -12.77 & 78.0 & 11.0 & 15.0 \\
\hline
\end{tabular}

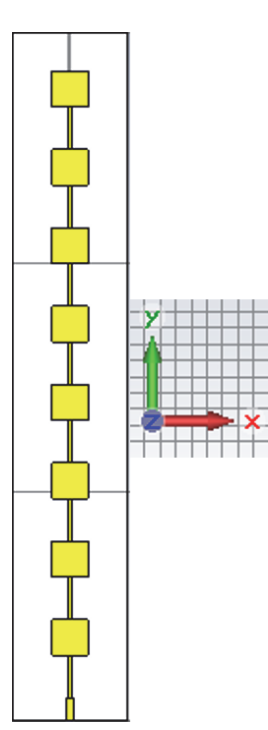

(a)

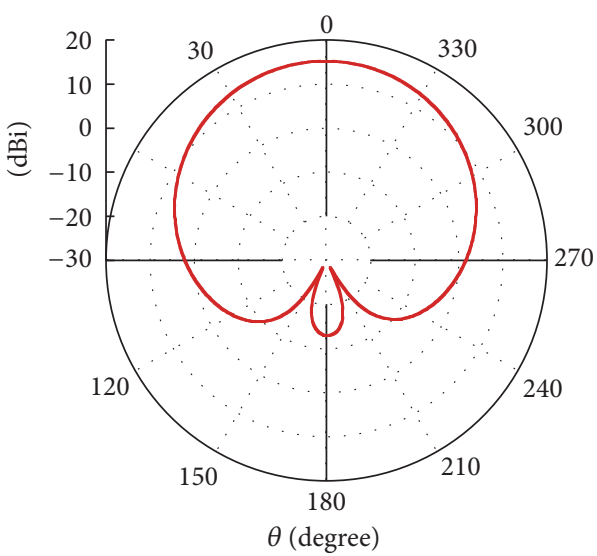

— Farfield directivity Abs $(\phi=0)$

(d)

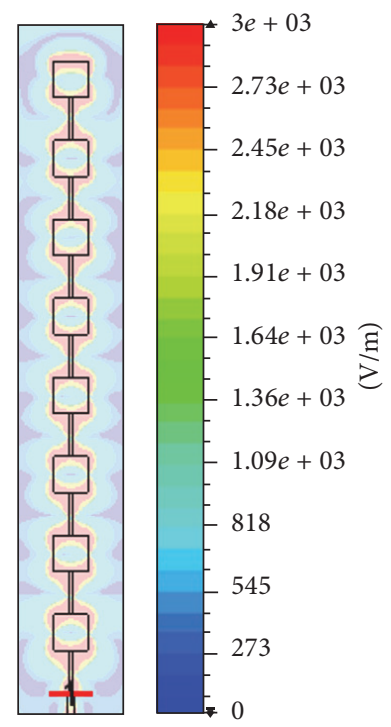

(b)

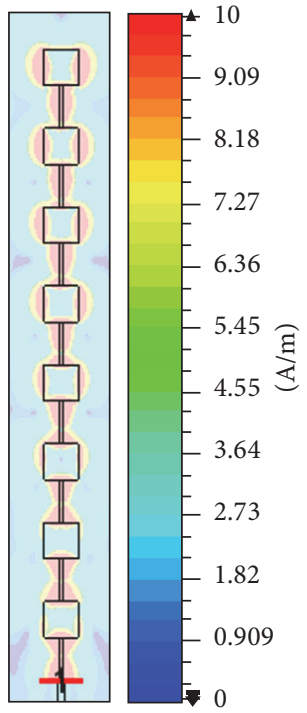

(c)

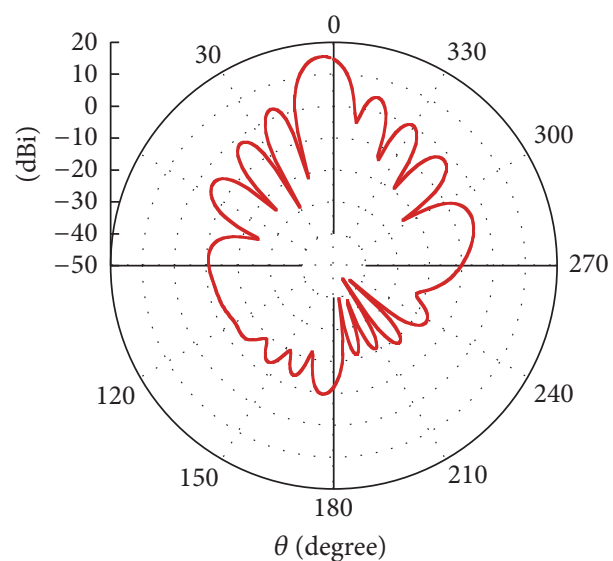

— Farfield directivity Abs $(\phi=90)$

(e)

FIGURE 5: Serial array with 8-patch geometry (a); $E_{z}$ field distribution (b) and $H_{z}$ field distribution (c) on the patch plane $(x-y)$; sagittal $(x-z)$ (d) and azimuthal $(y-z)(e)$ radiation patterns. 


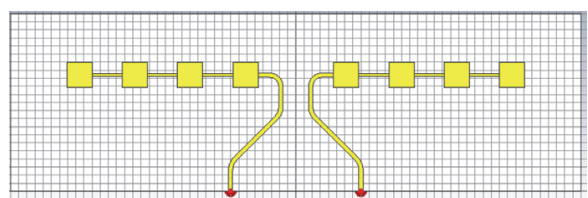

(a)

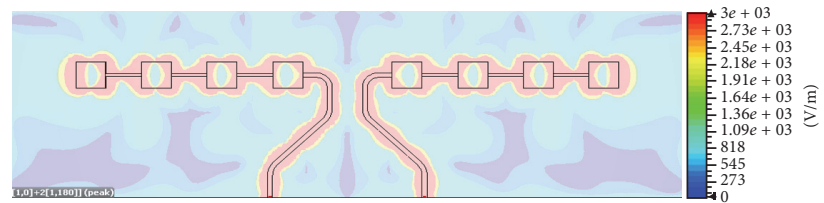

(b)

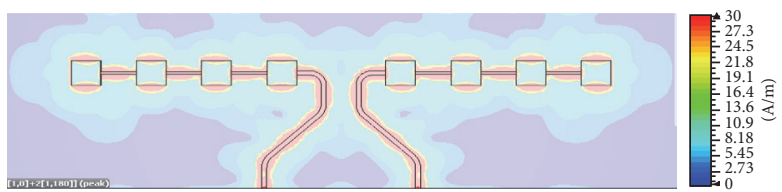

(c)

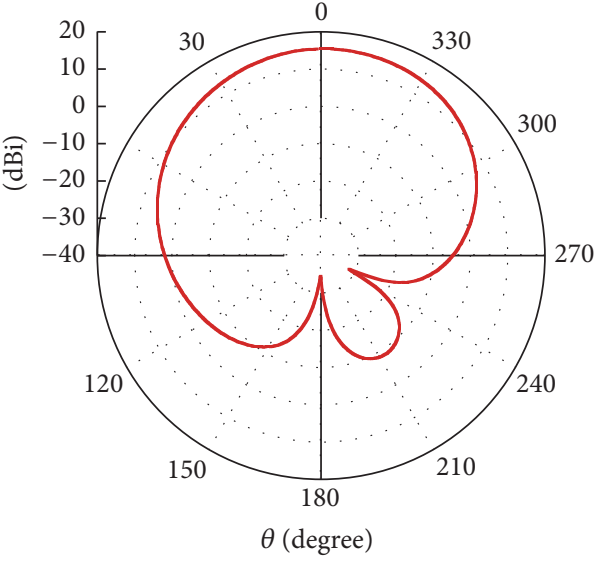

— Farfield directivity Abs $(\phi=90)$

(d)

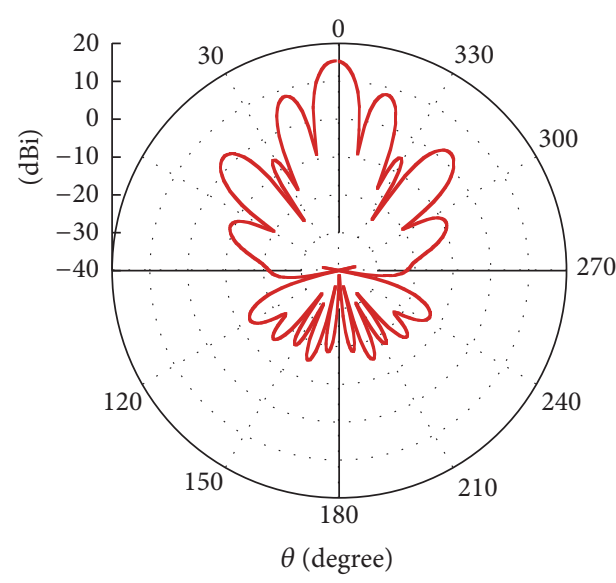

— Farfield directivity Abs $(\phi=0)$

(e)

Figure 6: Transmitting antenna geometry (a); $E_{z}$ field distribution (b) and $H_{z}$ field distribution (c) on the patch plane; sagittal (d) and azimuthal (e) radiation patterns.

is very easy to bend. This might cause malfunctions or, even worse, might break it.

The final step was to test the coaxial-to-microstrip transition realization by measuring with a network analyzer (PNA E8363C) the reflection coefficient at the coaxial port. The obtained results are reported in Figure 9. The figure shows, in the ISM band, a minimum in the reflection coefficient of $-17 \mathrm{~dB}$ very close to the theoretical value for a five-patch serial array (see Table 1). This result indicates that the designed transition does not reduce the expected performances of the serial array.

4.2. Antenna Realization. The same procedure described for the coaxial-to-microstrip transition realization has been followed for the antennas. Figure 10(a) shows the serial array with 8 patches, while Figures 10 (b) and 10 (c) show the transmitting and receiving antennas, respectively.

The reflection coefficients of the serial array with 8 patches and of the receiving antenna have been measured with the PNA E8363C and the obtained results are reported in Figures 11(a) and 11(b), respectively.
The measured reflection coefficients show for both antennas good matching in the ISM band around $24.125 \mathrm{GHz}$ with values below $-10 \mathrm{~dB}$.

\section{Radar Test}

The whole system has been assembled as depicted in Figure 1 (a). The sawtooth signal with a repetition rate of $300 \mathrm{~Hz}$ has been generated by using the NI DAQ USB 6251 card controlled by a virtual instrument developed in the LabVIEW environment. The same DAQ has been used for the acquisition of the output signal and for the signal frequency extraction by fast Fourier transform (FFT). Figure 12 shows the obtained FFT when a metallic panel $(30 \times 50 \mathrm{~cm})$ is placed $1.4 \mathrm{~m}$ far from the radar antennas. As expected from (1), $f_{\text {Delay }}$ $=750 \mathrm{~Hz}$ has been obtained. Altogether, the system was able to identify targets up to the requested distance of $5 \mathrm{~m}$.

\section{Conclusions}

In this paper, the design, realization, and test of the transmitting and receiving antennas for an FMCW radar operating 


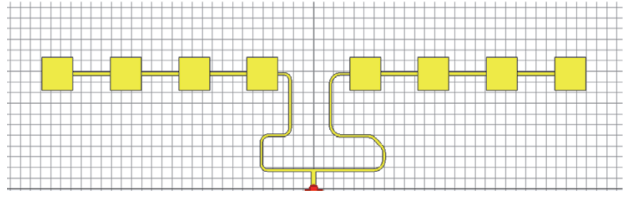

(a)

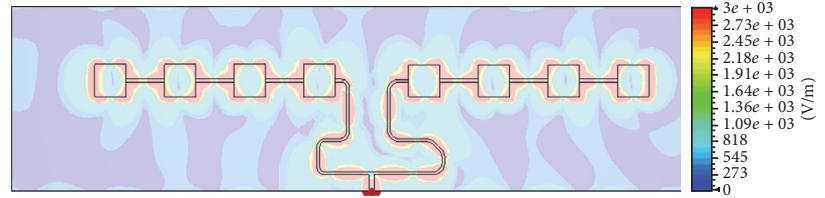

(b)

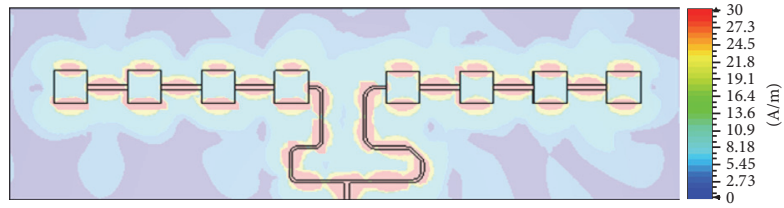

(c)

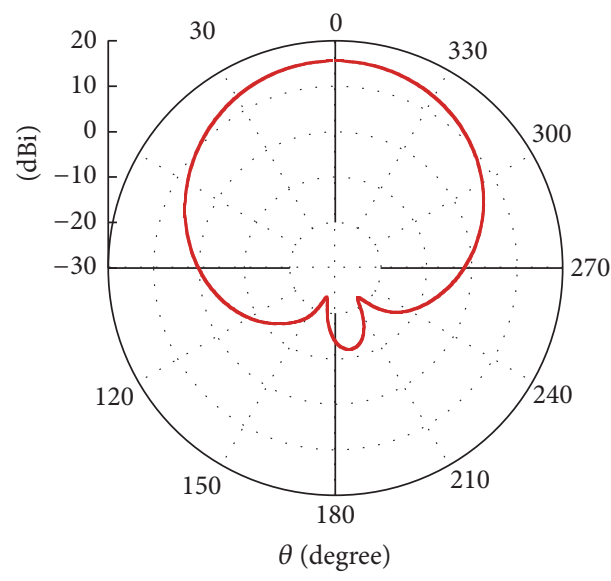

— Farfield directivity Abs $(\phi=90)$

(d)

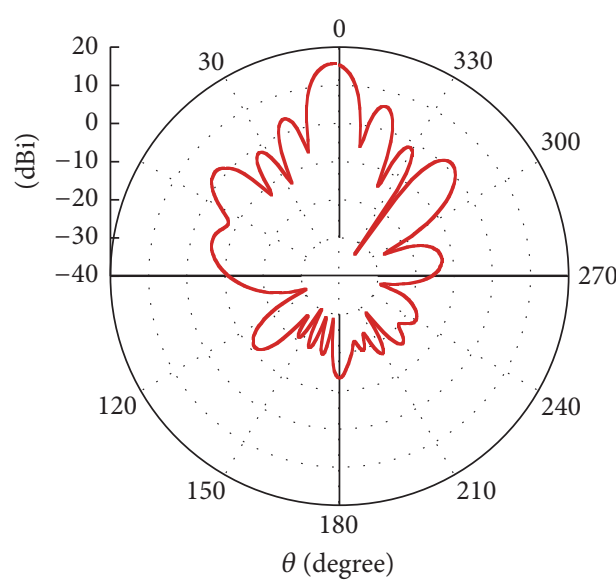

— Farfield directivity Abs $(\phi=0)$

(e)

FIGURE 7: Receiving antenna geometry (a); $E_{z}$ field distribution (b) and $H_{z}$ field distribution (c) on the patch plane; sagittal (d) and azimuthal (e) radiation patterns.

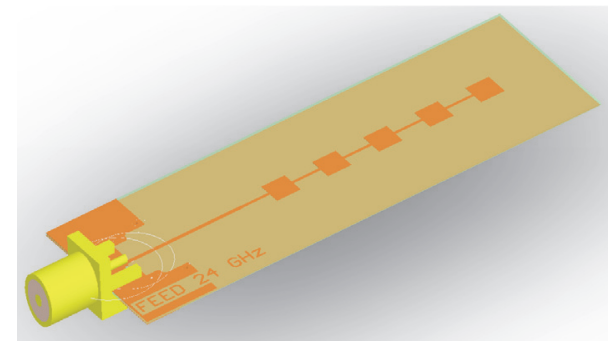

(a)

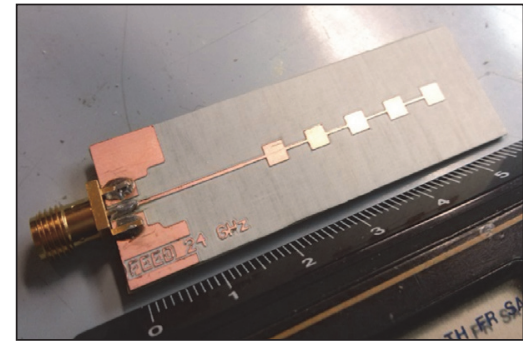

(b)

FIGURE 8: Model of the $24 \mathrm{GHz}$ coaxial-to-microstrip transition (a) and realized structure (b).

between 24 and $24.250 \mathrm{GHz}$, to be housed in a white cane, have been performed.

The coaxial-to-microstrip transition necessary for feeding the antennas has been studied and a structure constituted by the cascade of three waveguides has been optimized. With respect to the transition proposed in [12], the one developed in this paper is simpler and more suitable to be realized on a large scale.

A serial array with 8 patches has been selected for the transmitting and receiving antennas. Exploiting the radar differential output and a Wilkinson power divider with $180^{\circ}$ phase difference, the serial array has been splitted into two 


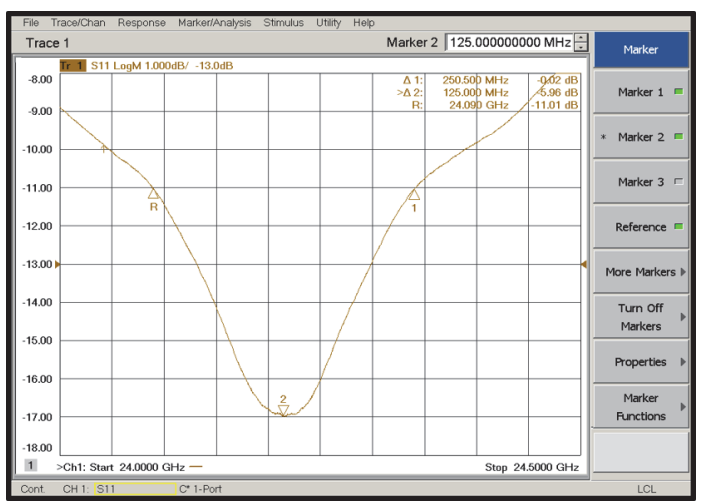

FIGURE 9: Measured reflection coefficient of the antenna depicted in Figure 8.

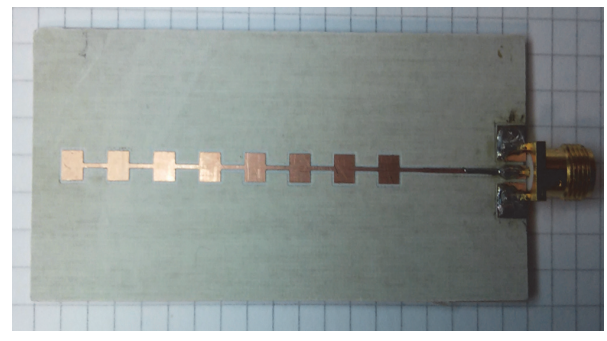

(a)

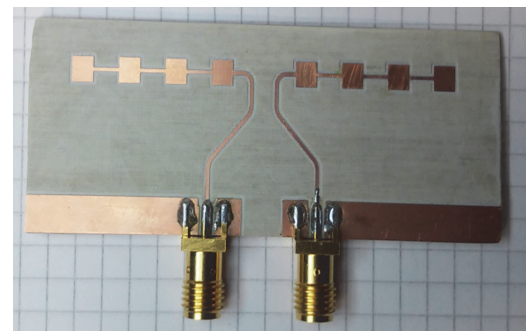

(b)

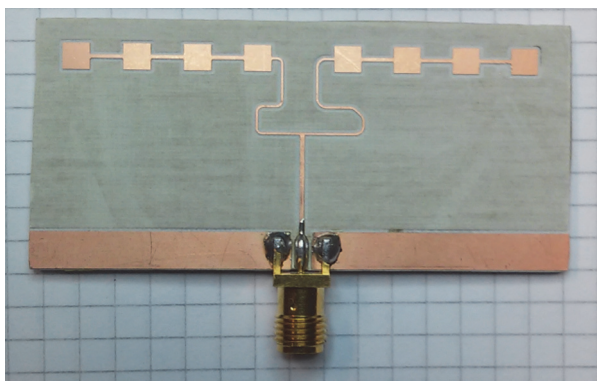

(c)

FIGURE 10: Serial array with 8 patches (a); transmitting (b) and receiving (c) antennas.

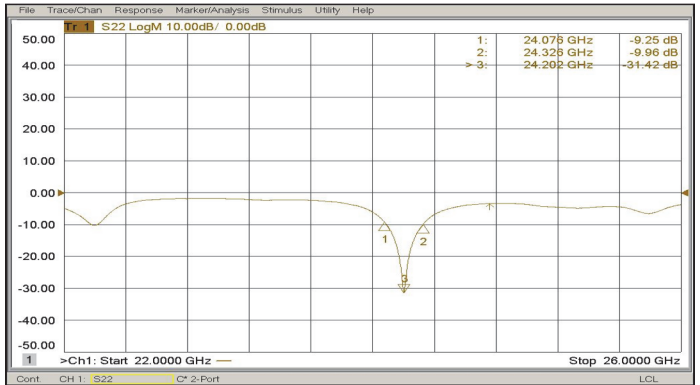

(a)

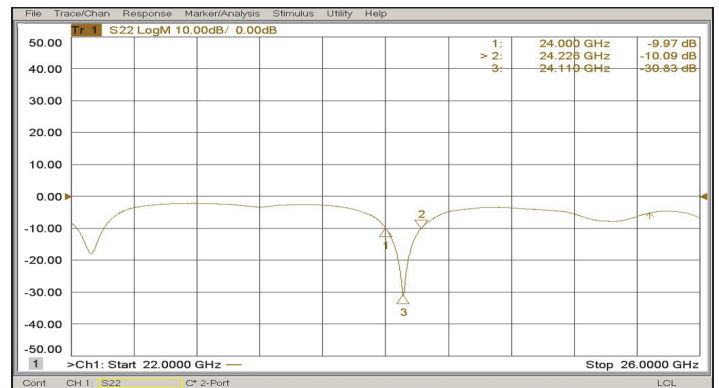

(b)

FIGURE 11: Measured reflection coefficient of the serial array with 8 patches (a) and of the receiving antenna (b). 


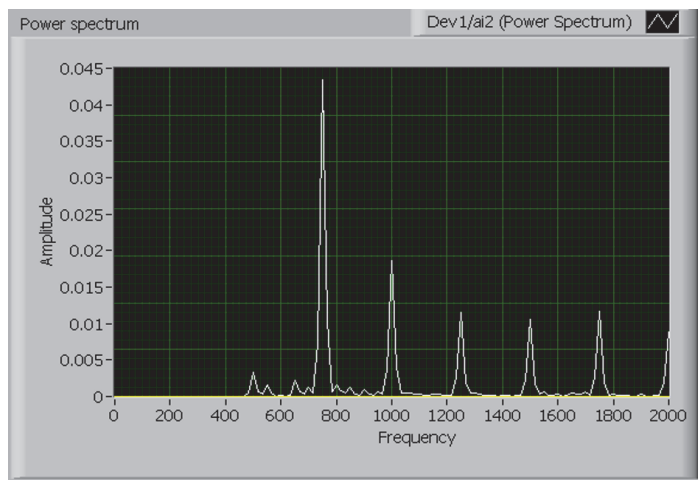

FIGURE 12: FFT of the radar output for a metallic panel $1.4 \mathrm{~m}$ far from the radar antenna.

serial arrays with four patches, making the structure more symmetrical and easier to be realized.

The realized structures showed reflection coefficients lower than $-10 \mathrm{~dB}$ in the ISM band. When the antennas were attached to an FMCW radar board, the radar was able to record reflections from a metallic panel up to a $5 \mathrm{~m}$ distance.

\section{Competing Interests}

The authors declare that they have no competing interests.

\section{References}

[1] C. Corcoran, G. Douglas, S. Pavey, A. Fielding, M. McLinden, and S. McCall, "Network 1000: the changing needs and circumstances of visually-impaired people: project overview," The British Journal of Visual Impairment, vol. 22, no. 3, pp. 93-100, 2004.

[2] B. B. Blasch, W. R. Wiener, and R. L. Welsh, Foundations of Orientation and Mobility, 2nd edition, 1997.

[3] U. R. Roentgen, G. J. Gelderblom, M. Soede, and L. P. de Witte, "Inventory of electronic mobility aids for persons with visual impairments: a literature review," Journal of Visual Impairment \& Blindness, vol. 102, no. 11, pp. 702-724, 2008.

[4] D. Dakopoulos and N. G. Bourbakis, "Wearable obstacle avoidance electronic travel aids for blind: a survey," IEEE Transactions on Systems, Man and Cybernetics Part C: Applications and Reviews, vol. 40, no. 1, pp. 25-35, 2010.

[5] L. Scalise, V. M. Primiani, P. Russo et al., "Experimental investigation of electromagnetic obstacle detection for visually impaired users: a comparison with ultrasonic sensing," IEEE Transactions on Instrumentation and Measurement, vol. 61, no. 11, pp. 3047-3057, 2012.

[6] S. Linz, G. Vinci, S. Mann et al., "A compact, versatile six-port radar module for industrial and medical applications," Journal of Electrical and Computer Engineering, vol. 2013, Article ID 382913, 10 pages, 2013.

[7] P. Bernardi, R. Cicchetti, S. Pisa, E. Pittella, E. Piuzzi, and O. Testa, "Design, realization, and test of a UWB radar sensor for breath activity monitoring," IEEE Sensors Journal, vol. 14, no. 2, pp. 584-596, 2014.

[8] E. Pittella, P. Bernardi, M. Cavagnaro, S. Pisa, and E. Piuzzi, "Design of UWB antennas to monitor cardiac activity," The
Applied Computational Electromagnetics, vol. 26, no. 4, pp. 267274, 2011.

[9] G. A. Zito, E. M. Staderini, and S. Pisa, "A twin spiral planar antenna for UWB medical radars," International Journal of Antennas and Propagation, vol. 2013, Article ID 684185, 6 pages, 2013.

[10] A. G. Stove, "Linear FMCW radar techniques," IEE Proceedings Part F: Radar and Signal Processing, vol. 139, no. 5, pp. 343-350, 1992.

[11] E. L. Holzman, Essentials of RF and Microwave Grounding, Artech House, Norwood, Mass, USA, 2006.

[12] L. Zhu and K. L. Melde, "On-wafer measurement of microstripbased circuits with a broadband vialess transition," IEEE Transactions on Advanced Packaging, vol. 29, no. 3, pp. 654-659, 2006.

[13] C. A. Balanis, Antenna Theory Analysis and Design, John Wiley \& Sons, New York, NY, USA, 1997. 


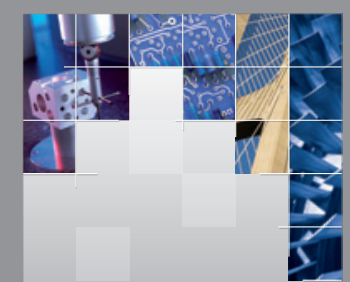

\section{Enfincering}
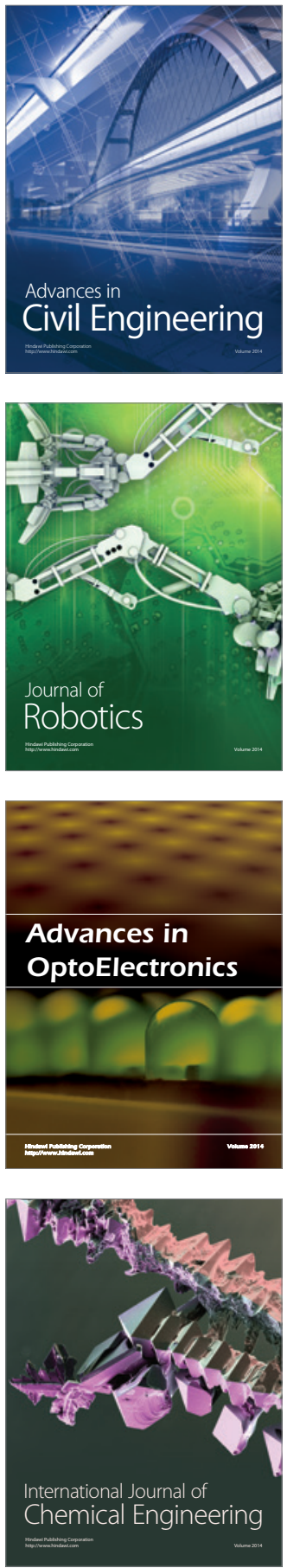

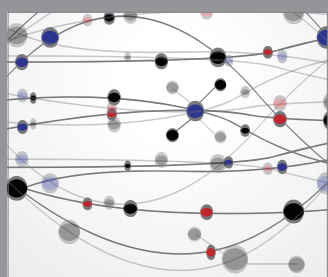

The Scientific World Journal

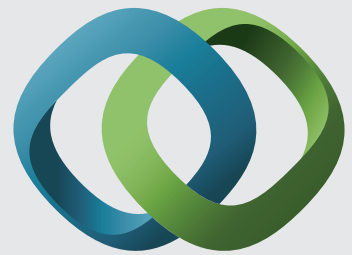

\section{Hindawi}

Submit your manuscripts at

http://www.hindawi.com
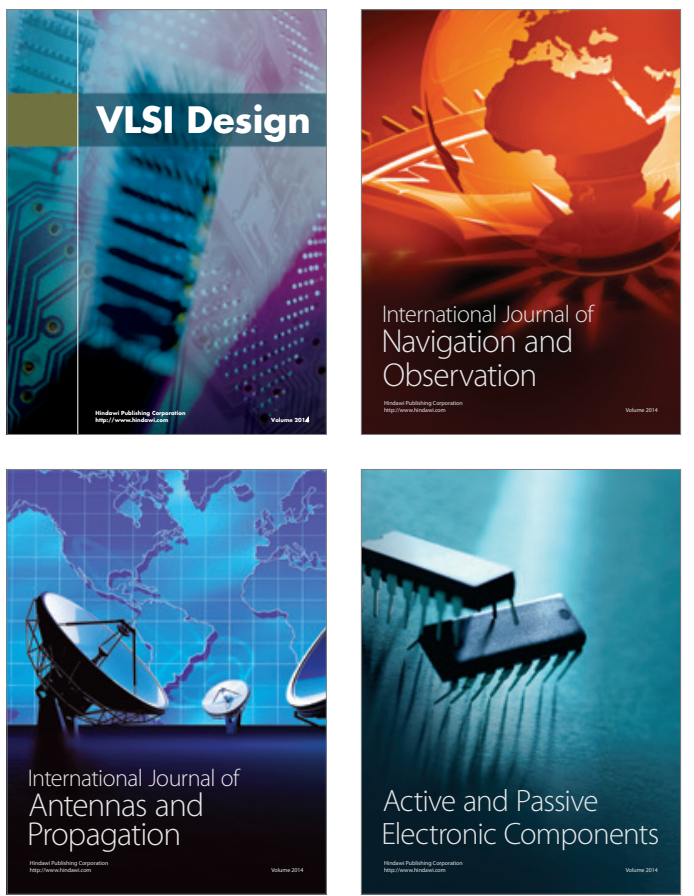
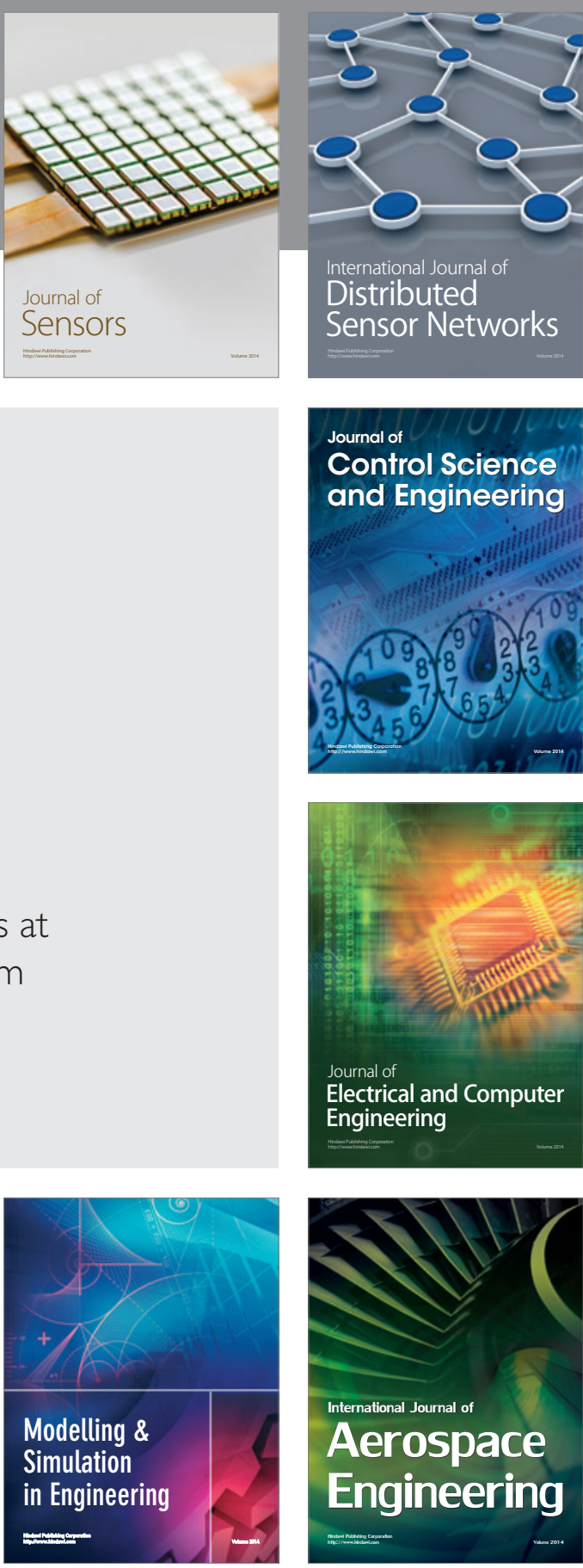

International Journal of

Distributed

Sensor Networks

Journal of

Control Science

and Engineering
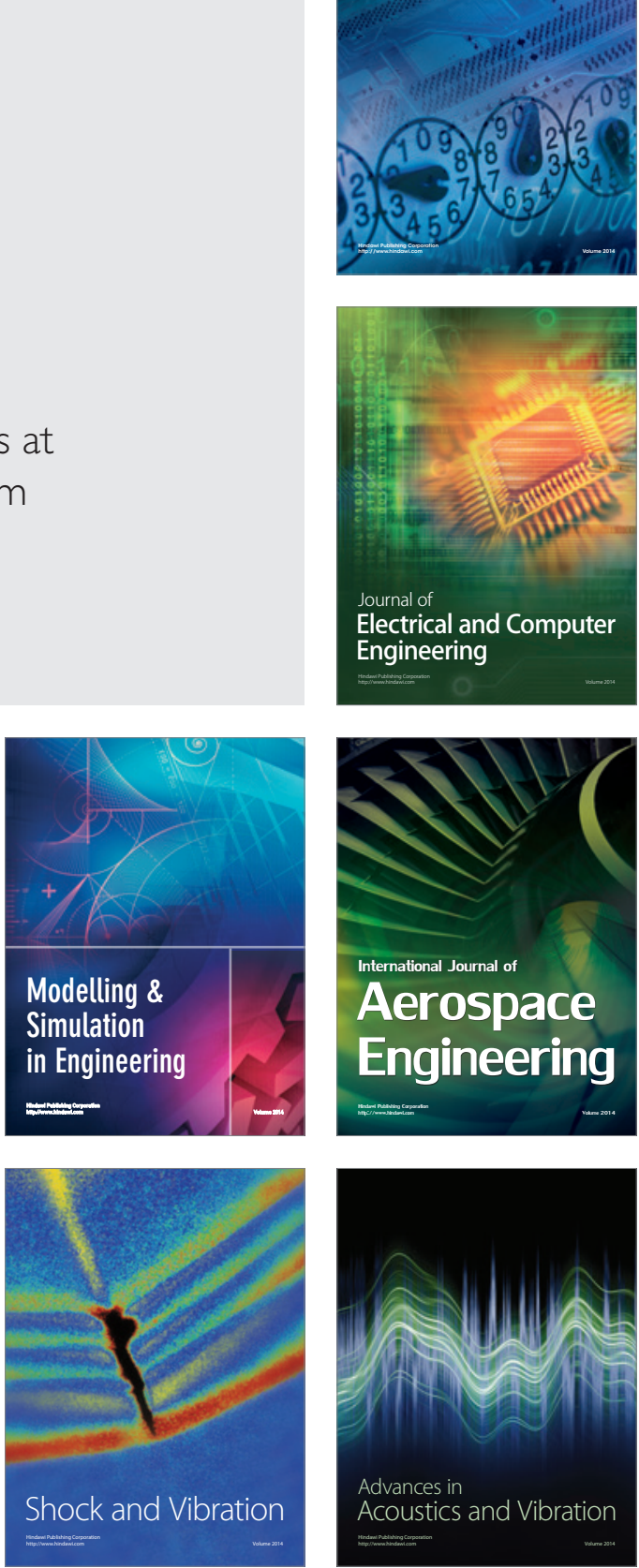